\title{
Reducing Computational Overhead by Improving the CRI and IRI Implication Step
}

\author{
Thoai Phu Vo and Joy Iong-Zong Chen \\ Department of Electrical Engineering, Dayeh University, No. 168, University Road, Changhua 51591, Taiwan \\ Correspondence should be addressed to Thoai Phu Vo; thoaiphuvo@yahoo.com
}

Received 18 December 2014; Accepted 5 March 2015

Academic Editor: Lifeng Ma

Copyright (C) 2015 T. P. Vo and J. I.-Z. Chen. This is an open access article distributed under the Creative Commons Attribution License, which permits unrestricted use, distribution, and reproduction in any medium, provided the original work is properly cited.

In conventional SISO fuzzy expert systems ( $n$-element input, $m$-element output), the implication step requires the $O(n \times m)$ operations using compositional rule-based inference (CRI) and individual rule-based inference (IRI). However, this introduces excessive complexity. This paper proposes two methods, sort compositional rule-based inference (SCRI) and sort individual rulebased inference (SIRI) aiming at reducing both temporal and spatial complexity by changing the operation of the implication step to $O\left((n+m) \log _{2}(n+m)\right)$. We also propose a divide-and-conquer technique, called Quicksort, to verify the accuracy of SCRI and SIRI algorithms deployment to easily outperform the CRI and IRI methods.

\section{Introduction}

The inference compositional rule (CRI) was first proposed by Zadeh [1] to solve the fuzzy modus ponens (FMP) and fuzzy modus tollens (FMT) models. Later Dubois and Prade [2] introduced two approaches to present the inference of a set of parallel rules for solving the local inference approach, known as IRI, and the global inference approach, which is known as CRI. Many researches have been conducted on CRI and IRI. The first topic is "aggregation operator" [3] introducing two methods to deal with aggregation operator issues. The first infer then aggregate (FITA) is known as IRI, and the first aggregate then infer (FATI) is known as CRI. They attempt to find an operator for CRI and IRI aggregation. Assilian [4] used the max-min method. A "min" operator is selected as a conjunction in the rule premise and the implication function while a "max" operator is used for aggregation. Dubois [5] used a "min" operator for aggregation in decision-making. To reduce the computational time, the authors $[6,7]$ replaced a MISO fuzzy rule with an equivalent collection of SISO rules with two kinds of aggregation operators.

The second topic involves fuzzy logic application in many fields using the CRI method, the second topic. Mamdani [8] applied fuzzy logic in designing controllers for industrial plants. Reference [9] applied an adaptive fuzzy inference scheme to stock data and showed that the adaptive fuzzy rule-based schemes provide clean decision to buy, hold, and sale. Reference [10] proposed a fuzzy interval reasoning (FIR) and [11] introduced a fuzzy logic controller to serve web shopping. The third topic is new algorithms based on the CRI context. Reference [12] presented a new feedback method based on CRI (FBCRI) to improve the robustness of CRI fuzzy reasoning methods. Reference [13] introduced a CMI (compatibility modification inference), which is reduced by CRI. References [14-21] developed the Triple I method, based on CRI imperfections. Accordingly, [14] proposed the full implication Triple I method (a hybrid method based on implication and composition), designed as a supplement for the CRI method. Reference [15] proposed the Triple I FMP and FMT for generalized $\alpha$-triple I method. An investigation into a formalized Triple I method is reported in [16] where the authors tried to set a sound logical foundation for the unified TI algorithms. Accordingly, other researchers developed new implications, the fourth topic. Reference [22] proposed a new implication: "nilpotent minimum implication."

Other researchers studied Mamdani and Sugeno type fuzzy model applications, the fifth topic. Reference [23] showed the advantage of Sugeno type fuzzy inference system 
in that it can be integrated with neural networks and genetic algorithms or other optimization techniques. In [24] the Mamdani and Sugeno models are used to compute the resonant frequency of rectangular microstrip antennas (MSAs) with thin and thick substrates. Finally, [25] implemented a supervised learning method based on membership function training in the Mamdani fuzzy model context. Specifically, the autozoom function of a digital camera is modeled adopting the Mamdani technique.

We have shown five research topics to view that the improvement of CRI and IRI has never been done. SCRI and SIRI are used to reduce the computational time and space complexity of CRI and IRI in this research. Suppose that there are $r$ rules in a fuzzy expert system rule base and they are defined as

$$
\text { If } x \text { is } A^{k} \text {, then } y \text { is } B^{k} \text {, }
$$

where $A^{k}$ and $B^{k}, k=1,2, \ldots, r$, are fuzzy sets defined in the universe of discourses $V$ and $W$, respectively.

Two basic methods are used to obtain a meaningful inference for a given observation $A^{\prime}$. The first method, CRI, includes three steps: (1) implication, (2) aggregation, and (3) composition. The second method, IRI, includes three steps: (1) implication, (2) composition, and (3) aggregation. The two methods include the same implication step, which includes two substeps: cylindrical extension and calculating relational matrix using a space of $O(n \times m)$ and an operation of $O(n \times m)$. The main contributions of this paper are as follows.

(i) This paper clearly showed the advantages of CRI comparing to IRI in SISO fuzzy system.

(ii) This paper proposes two methods, sort compositional rule-based inference (SCRI) and sort individual rulebased inference (SIRI), aiming at reducing the operation of the implication step from $O(n \times m)$ to $O((n+$ $\left.m) \log _{2}(n+m)\right)$.

\section{Multiple Fuzzy Reasoning Schemes}

2.1. Quicksort. This study adopted a method referred to as Quicksort, a divide-and-conquer method, which proceeds through four steps: (1) arbitrarily select an element in the array (the pivot), (2) divide the array into two subarrays of uneven size (the partition phase), (3) sort the subarrays recursively, and (4) position the pivot between the two sorted subarrays to obtain the final sorted array. The Quicksort performance depends on the pivot elements in the array, as outlined below.

2.1.1. The Worst Case. The worst case scenario occurs when the pivot element is the largest or smallest element in the array. In Figure 1, the pivot element is the smallest element in the array. The total number $C(l)$ of comparisons performed by Quicksort in sorting an array of size $l$ is calculated as

$$
C(l)=l+C(k-1)+C(l-k),
$$

where $C(0)=C(1)=0$ is assumed, $k$ is the final position of pivot element, and $C(l)$ is the total number of comparisons

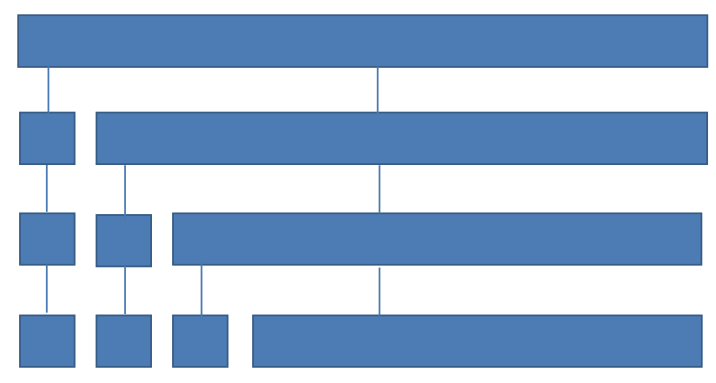

FIGURE 1: The worst recursion tree case for Quicksort.

performed by Quicksort in sorting an array of size $l$, which is the number of comparisons that any partitioning algorithm can perform. Accordingly, the reason is that every element other than the pivot must be compared to the pivot. Moreover, $C(k-1)$ in (2) is the number of comparisons of left subarray by recursive call to Quicksort, and $C(l-k)$ is the number of comparisons of right subarray by recursive call to Quicksort. In the worst case, the parameter, $k$, can be assigned as 1 or $l$. For example, when assuming that $k$ is equivalent to 1 , then (2) becomes

$$
\begin{aligned}
C(l) & =l+C(l-1) \\
& =l+l-1+C(l-2) \\
& =\frac{l(l+1)}{2}-1,
\end{aligned}
$$

and while $l \gg 1$, the last equation becomes

$$
C(l) \approx \frac{l^{2}}{2} \in O\left(l^{2}\right) .
$$

Several appropriate pivot selection methods have been devised: (a) first element, (b) last element, (c) median-ofthree elements, and (d) random element. It is worth noting that Quicksort generally chooses a pivot at random, which greatly reduces the likelihood for the worst case occurring and tends to work very well. The other means of avoiding the worst case is the median-of-three elements.

2.1.2. The Best Case. As shown in Figure 2, the best case has the pivot element with the middle element in the array. To know the total number $C(l)$ for the best case, $k=(l+1) / 2$ is considered and the results from (2) are obtained as $C(l)=$ $l+2 C((l-1) / 2)$. It can be approximately examined as

$$
\begin{aligned}
C(l) & =l+2 C\left(\frac{l}{2}\right) \\
& =l+2\left(2 C\left(\frac{l}{4}\right)+\frac{l}{2}\right) \\
& =2 l+2^{2} C\left(\frac{l}{2^{2}}\right) \\
& =\alpha l+2^{\alpha} C\left(\frac{l}{2^{\alpha}}\right) .
\end{aligned}
$$




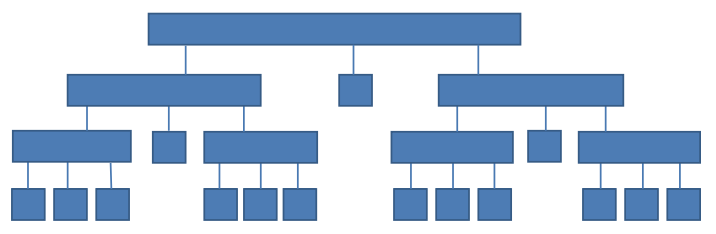

FIGURE 2: The best recursion tree case for Quicksort.

It should be noted that, after the $\alpha$ th calculation step, this recurrence will continue only until $l=2^{\alpha}$ (otherwise we have $l / 2^{\alpha}<1$ ), that is, until $\alpha=\log _{2} l$. Thus, by putting $\alpha=\log _{2} l$ into the previous equation, the equation can be obtained as follows:

$$
C(l)=l \cdot \log _{2} l
$$

2.1.3. The Average Case. The average case occurs when the pivot element is near the middle element in the array (random pivot). As shown in Figure 3, random pivots are used to generate the average case in the recursion tree for Quicksort.

In summary, three cases are used to understand the calculation complexity for the Quicksort algorithm. Currently, there is an axiom that could be decided.

Theorem 1. The average case in Quicksort has the expected run time of $\mathrm{O}\left(l \cdot \log _{2} l\right)$, with $l$ as the number of elements in the array.

Proof. The Harmonic numbers are given as

$$
H_{l}=\sum_{i=1}^{l} \frac{1}{i} \approx \ln l .
$$

It is assumed that each possible pivot $k$ is selected with equal probability, therefore, (2) can be averaged over all $k$, that is given as

$$
\begin{aligned}
C(l) & =\left(\frac{1}{l}\right) \sum_{k=1}^{l}(l+C(k-1)+C(l-k)) \\
& =l+\left(\frac{1}{l}\right) \sum_{k=1}^{l} C(k-1)+\left(\frac{1}{l}\right) \sum_{k=1}^{l} C(l-k) .
\end{aligned}
$$

By substituting $i=k-1$ and $i=l-k$ into the last equation, the averaged equation can be determined as

$$
\begin{aligned}
& \sum_{k=1}^{l} C(k-1)=\sum_{i=0}^{l-1} C(i), \\
& \sum_{k=1}^{l} C(l-k)=\sum_{i=0}^{l-1} C(i) .
\end{aligned}
$$

If the last results are taken into account, the developed recurrence becomes (see Appendix A)

$$
\begin{aligned}
& C(l)=l+\left(\frac{2}{l}\right) \sum_{i=0}^{l-1} C(i) \\
& \Longleftrightarrow l C(l)=(l+1) C(l-1)+2 l-1 .
\end{aligned}
$$

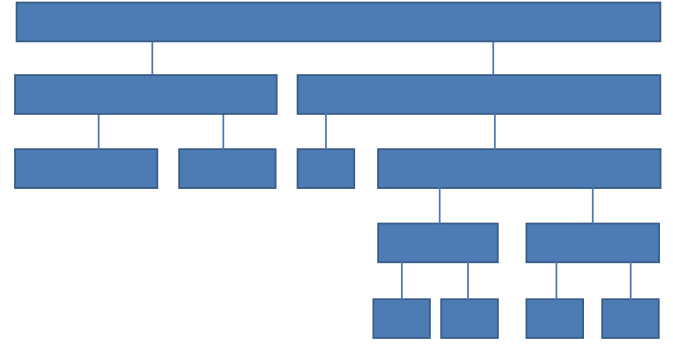

FIGURE 3: The average recursion tree case for Quicksort.

By dividing the last equation with $l(l+1)$, it is written as

$$
\begin{aligned}
& \frac{C(l)}{(l+1)}=\frac{C(l-1)}{l}+\frac{(2 l-1)}{(l(l+1))} \\
& \Longleftrightarrow \frac{C(l)}{(l+1)}=\frac{C(l-1)}{l}+\frac{2}{l} .
\end{aligned}
$$

After the assignment of $D(l)=C(l) /(l+1)$, the recurrence becomes as follows:

$$
\begin{aligned}
D(l) & =D(l-1)+\frac{2}{l} \\
& =D(l-2)+\frac{2}{(l-1)}+\frac{2}{l} \\
& =\cdots \\
& =D(1)+\frac{2}{2}+\frac{2}{3}+\cdots+\frac{2}{(l-2)}+\frac{2}{(l-1)}+\frac{2}{l} \\
& \approx 2 \ln (l) \\
& \approx 1.39 \log _{2}(l),
\end{aligned}
$$

where the $D(1)=0$ is considered in the previous equation. In the advanced method, the recurrence is able to be performed as

$$
C(l)=(l+1) D(l) \approx 1.39(l+1) \log _{2}(l)
$$

moreover, when $l \gg 1$ is considered, then

$$
C(l) \approx 1.39 l \log _{2}(l) \in O\left(l \log _{2} l\right) \text {. }
$$

The worst case has the biggest or smallest pivot element in the Quicksort array, as discussed in Section 2.1.1. Table 1 shows that merge sort and Quicksort have the same time complexity of $O\left(l \log _{2} l\right)$. Quicksort is the best one of the four algorithms because its space overhead is only $O(1)$.

2.2. SISO Multirule System. Suppose that $r$ rules exist in the fuzzy expert system rule base as follows:

$$
\text { If } x \text { is } A^{k} \text {, then } y \text { is } B^{k} \text {, }
$$

where $A^{k}$ and $B^{k}, k=1,2, \ldots, r$, are fuzzy sets defined in the universe of discourses $V$ and $W$, respectively. 
TABLE 1: Different sorting algorithms.

\begin{tabular}{lccc}
\hline $\begin{array}{l}\text { Sorting } \\
\text { algorithm }\end{array}$ & Worst case time & $\begin{array}{c}\text { Average case } \\
\text { time }\end{array}$ & $\begin{array}{c}\text { Space } \\
\text { overhead }\end{array}$ \\
\hline Bubble sort & $O\left(l^{2}\right)$ & $O\left(l^{2}\right)$ & $O(1)$ \\
Insertion sort & $O\left(l^{2}\right)$ & $O\left(l^{2}\right)$ & $O(1)$ \\
Merge sort & $O\left(l \log _{2} l\right)$ & $O\left(l \log _{2} l\right)$ & $O(l)$ \\
Quicksort & $O\left(l^{2}\right)$ & $O\left(l \log _{2} l\right)$ & $O(1)$ \\
\hline
\end{tabular}

\subsubsection{The Comparison of CRI and IRI}

(1) CRI and IRI Schemes. In order to achieve a meaningful inference result based on Zadeh's compositional rule of inference (CRI) [2], for a given system observation there are two basic methods adopted as important illustrations. The first one is called CRI or FATI (first aggregate then infer) [3]. In this first method, for a given observation of $A^{\prime}$, the implication is the first step performed to calculate the relational matrix given as

$$
R^{k}=I\left(A^{k}, B^{k}\right)
$$

where $R^{k}$ is the fuzzy implication relation for rule $k$ and $I$ denotes the implication method. All of the rules are then aggregated by structuring an overall fuzzy relation $R$ which is the combination of all relational matrixes and written as

$$
R=\bigcup_{k=1}^{r} R^{k},
$$

where $R$ indicates an overall fuzzy relation and $U$ is defined as a combination operator of all the fuzzy implication relations. The last equation is with a final result based on a given observation $A^{\prime}$ and shown as

$$
B^{\prime}=A^{\prime} \circ R
$$

where $\circ$ represents composition within the CRI context and $B^{\prime}$ is an inference result.

The second one is called individual rule-based inference (IRI) or FITA (first infer then aggregate) [3]. For a given observation $A^{\prime}$, we first perform the implication step to calculate the relational matrix as follows:

$$
R^{k}=I\left(A^{k}, B^{k}\right)
$$

where $R^{k}$ denotes the fuzzy implication relation for rule $k$ and $I$ is the implication method.

A result for every rule based on a given observation $A^{\prime}$ is then inferred as

$$
B^{k^{\prime}}=A^{\prime} \circ R^{k}
$$

where $B^{k^{\prime}}$ is the inference result based on rule $k$. Finally, all of these intermediate results are combined and determined as

$$
B^{\prime}=\bigcup_{k=1}^{r} B^{k^{\prime}} .
$$

Table 2 showed the CRI and IRI schemes.
TABLE 2: CRI and IRI schemes.

\begin{tabular}{lcc}
\hline & CRI & IRI \\
\hline Step 1 & Implication & Implication \\
Step 2 & Aggregation & Composition \\
Step 3 & Composition & Aggregation \\
\hline
\end{tabular}

TABLE 3: CRI and IRI time complexity comparison.

\begin{tabular}{lcc}
\hline & $\begin{array}{c}\text { CRI } \\
\text { time complexity } \\
\text { (operations) }\end{array}$ & $\begin{array}{c}\text { IRI } \\
\text { time complexity } \\
\text { (operations) }\end{array}$ \\
\hline (1) Implication & $r n m$ & $r n m$ \\
(2) Aggregation (CRI) & $(r-1) n m$ & \\
(3) Composition $\quad$ Intersection & $n m$ & $r n m$ \\
$\quad$ Projection & $(n-1) m$ & $r(n-1) m$ \\
(4) Aggregation (IRI) & & $(r-1) m$ \\
\hline Total time & $r n m+(r-1) n m$ & $r n m+r n m$ \\
& $+n m+(n-1) m$ & $+r(n-1) m$ \\
& & $+(r-1) m$ \\
\hline
\end{tabular}

(2) Time Complexity. In Table 3, $r$ represents the number of rules, $n$ is the number of elements in the input, and $m$ indicates the number of elements in the output. Assume that minimum and maximum operations require the same effort. Applying (16) to each rule, every $R^{k}$ requires $n m$ operations and results in the implication step with $r$ rules requires $\mathrm{rnm}$ operations. Based on (17), the aggregation of $r$ relation matrixes requires $(r-1) n m$ operations. The CRI composition requires $\mathrm{nm}$ operations for intersection and $(n-1) m$ operations for projection. Both intersection and projection are calculated using (18). To infer a conclusion from a SISO $r$ rules with CRI method, the total time can be easily determined as

$$
t_{\mathrm{CRI}}=r n m+(r-1) n m+n m+(n-1) m,
$$

where $t_{\mathrm{CRI}}$ denotes the CRI method time complexity. Using the result for every rule based on a given observation shown in (19), the IRI implication requires $n m$ operations for every relational matrix $R^{k}$, and so with $r$ relational matrixes it is just $\mathrm{rnm}$ operations. The IRI composition requires $\mathrm{rnm}$ operations for intersection and $r(n-1) m$ operations for projection. Both intersection and projection are calculated using (20).

Adopting (21), the IRI aggregation of $r$ conclusions $B^{k^{\prime}}$ will obtain $(r-1) m$ operations. A conclusion from SISO $r$ rules can be derived with the IRI method, with the total time measured as

$$
t_{\mathrm{IRI}}=r n m+r n m+r(n-1) m+(r-1) m,
$$

where $t_{\text {IRI }}$ is the IRI method time complexity.

The advantage of CRI compares to IRI in SISO rules expressed analytically in terms of gain $G$ as

$$
G=\frac{t_{\mathrm{IRI}}}{t_{\mathrm{CRI}}} .
$$


TABLE 4: CRI and IRI space complexity comparison.

\begin{tabular}{lcc}
\hline & $\begin{array}{c}\text { CRI } \\
\text { space complexity } \\
\text { (cells) }\end{array}$ & $\begin{array}{c}\text { IRI } \\
\text { space } \\
\text { complexity } \\
\text { (cells) }\end{array}$ \\
\hline (1) Implication & $\mathrm{rnm}$ & $\mathrm{rnm}$ \\
(2) Aggregation (CRI) & $n m$ & \\
(3) Composition & $\mathrm{mm}$ & $\mathrm{rnm}$ \\
$\quad$ Intersection & $\mathrm{m}$ & $\mathrm{rm}$ \\
(4) Aggregection & $\mathrm{rnm}$ & $\mathrm{m}$ \\
The biggest space of all steps & & $\mathrm{rnm}$ \\
\hline
\end{tabular}

By substituting (22) and (23) into the last equation, the gain becomes

$$
\begin{aligned}
G & =\frac{t_{\text {IRI }}}{t_{\mathrm{CRI}}} \\
& =\frac{2 r n m+r(n-1) m+(r-1) m}{r n m+(r-1) n m+n m+(n-1) m} \\
& =\frac{3 r n-1}{2 r n+n-1} \\
& \approx \frac{3}{2}>1 .
\end{aligned}
$$

The formula shows that gain $G$ is always bigger than 1 . We pointed out that the CRI method time complexity is less than the IRI method time complexity in the SISO rules expert system.

(3) Space Complexity. In Table 4, the same definition expressed in Table 3 is used for $r, n$, and $m$, assuming that every element requires one cell. Applying (16) to each rule, each $R^{k}$ requires $n m$ cells and results in the implication step with $r$ rules requires $r n m$ cells. Based on (17), it is clear that the aggregation of $r$ relation matrix requires $n m$ cells. Using (18), it is known that the composition of CRI requires $n m$ cells for intersection and $m$ cells for projection, respectively. In general, to infer a conclusion from SISO $r$ rules with the CRI method, the required space is determined using the biggest space from all steps and obtained as

$$
O(r n m) \text {. }
$$

To determine the implication that IRI requires $\mathrm{nm}$ cells for every relational matrix $R^{k}$ using (19), that is, with $r$, relational matrixes $\mathrm{rnm}$ cells are necessary. Based on (20), the IRI composition requires $\mathrm{rnm}$ cells for intersection and $\mathrm{rm}$ cells for projection, respectively. Therefore, with (21) the aggregation of $r$ conclusions $B^{k^{\prime}}$ requires $m$ cells. Hence, to infer a conclusion from SISO $r$ rules with the IRI method, the required space can be determined using the biggest space from all steps and written as

$$
O(r n m) \text {. }
$$

For the purpose of optimization, CRI combines step (1) (implication) with step (2) (aggregation); thus, it is easily understood that CRI needs a space of $\mathrm{O}(\mathrm{nm})$. IRI requires a space of $O(\mathrm{rnm})$ to store $r$ relational matrixes in the implication step. It is worth noting that the CRI method space complexity is less than the IRI method space complexity in the SISO rules expert system.

\subsubsection{CRI and SCRI}

(1) CRI. As mentioned in Section 2.2.1(1) the first CRI method step is the implication step, which includes two substeps. The first substep is cylindrical extension defined as

$$
A^{k} \_\mathrm{ex}=\left[\begin{array}{cccc}
a_{11}^{k} & a_{12}^{k} & \cdots & a_{1 m}^{k} \\
a_{21}^{k} & a_{22}^{k} & \cdots & a_{2 m}^{k} \\
\vdots & \vdots & \cdots & \vdots \\
a_{n 1}^{k} & a_{n 2}^{k} & \cdots & a_{n m}^{k}
\end{array}\right]_{n \times m},
$$

where $A^{k}$ _ex is extended by $A^{k}, a_{i j}^{k}=a_{i}^{k}$, with $A^{k}=$ $\left[a_{i}^{k}\right]_{i=1}^{n}, i=1,2, \ldots, n, j=1,2, \ldots, m, k=1,2, \ldots, r$ :

$$
B_{\text {_ex }}^{k}\left[\begin{array}{cccc}
b_{11}^{k} & b_{12}^{k} & \cdots & b_{1 m}^{k} \\
b_{21}^{k} & b_{22}^{k} & \cdots & b_{2 m}^{k} \\
\vdots & \vdots & \cdots & \vdots \\
b_{n 1}^{k} & b_{n 2}^{k} & \cdots & b_{n m}^{k}
\end{array}\right]_{n \times m},
$$

where $B^{k}$ _ex is extended by $B^{k}, b_{i j}^{k}=b_{j}^{k}$, with $B^{k}=\left[b_{j}^{k}\right]_{j=1}^{m}$, $i=1,2, \ldots, n, j=1,2, \ldots, m, k=1,2, \ldots, r$.

Filling all of the columns in the related matrix using the $X$-projection is cylindrical extension from $X$-projection which is shown in (28). Similarly, cylindrical extension from $Y$-projection means filling all of the rows in the relation matrix using the $Y$-projection as shown in (29). Calculating relation matrix is the second substep to be defined and it is written as

$$
\begin{aligned}
R^{k} & =\min \left(A^{k}{ }_{\text {ex }}, B^{k} \_\mathrm{ex}\right) \\
& =\left[\begin{array}{cccc}
q_{11}^{k} & q_{12}^{k} & \cdots & q_{1 m}^{k} \\
q_{21}^{k} & q_{22}^{k} & \cdots & q_{2 m}^{k} \\
\vdots & \vdots & \cdots & \vdots \\
q_{n 1}^{k} & q_{n 2}^{k} & \cdots & q_{n m}^{k}
\end{array}\right]_{n \times m},
\end{aligned}
$$

where $R^{k}$ is the relation matrix for rule $k$ and $q_{i j}^{k}=$ $\min \left(a_{i j}^{k}, b_{i j}^{k}\right), i=1,2, \ldots, n ; j=1,2, \ldots, m$. The implication is "min" as Mamdani fuzzy model. Aggregation, the second CRI method step, is defined as

$$
R=\bigcup_{k=1}^{r} R^{k},
$$


where $R$ is the overall relation matrix, which is calculated by combining all of the relation matrix $R^{k}$ with $k=1,2, \ldots, r$. Composition is the final CRI step and it is defined as (see Appendix B)

$$
\begin{aligned}
B^{\prime} & =A^{\prime} \circ R \\
& =\sup \left(\min \left(A^{\prime} \_ \text {ex, } R\right)\right) \\
& =\sup \left[\begin{array}{cccc}
b_{11}^{\prime} & b_{12}^{\prime} & \cdots & b_{1 m}^{\prime} \\
b_{21}^{\prime} & b_{22}^{\prime} & \cdots & b_{2 m}^{\prime} \\
\vdots & \vdots & \cdots & \vdots \\
b_{n 1}^{\prime} & b_{n 2}^{\prime} & \cdots & b_{n m}^{\prime}
\end{array}\right]_{n \times m}=\left[\begin{array}{llll}
b_{1}^{\prime} & b_{2}^{\prime} & \cdots & b_{m}^{\prime}
\end{array}\right],
\end{aligned}
$$

where $A^{\prime}$ _ex is extended by $A^{\prime}, B^{\prime}$ is the inference result which is calculated by compositing the observation $A^{\prime}$ with the overall relational matrix $R$, and o represents the composition method.

(2) SCRI (Sort CRI). In this subsection, the SCRI method is introduced. It includes three steps. The first step is implication which has two substeps. The first substep is sorting program and the second substep is getting relational matrix. The second step is aggregation defined as $R=\bigcup_{k=1}^{r} R^{k}$. Composition is the third step and given as $B^{\prime}(v)=A^{\prime} \circ R=$ sup $\min \left(A^{\prime}\right.$ _ex, $\left.R\right)$, where $A^{\prime}$ _ex is extended by $A^{\prime}$. Moreover, let us discuss the SCRI method implication step in detail. The SCRI implication has two substeps. The matrix $C^{k}$ is necessary for processing the first sorting program step, which can be expressed as

$$
C^{k}=\left[\begin{array}{cccccccc}
a_{1}^{k} & a_{2}^{k} & \cdots & a_{n}^{k} & b_{1}^{k} & b_{2}^{k} & \cdots & b_{m}^{k} \\
1.1 & 1.2 & \cdots & 1 . n & 2.1 & 2.2 & \cdots & 2 . m
\end{array}\right]_{2 \times(n+m)},
$$

where the elements in the first row of (33) are a combination of $A^{k}$ and $B^{k}$ with $A^{k}=\left[a_{1}^{k}, a_{2}^{k}, \ldots, a_{n}^{k}\right], B^{k}=\left[b_{1}^{k}, b_{2}^{k}, \ldots, b_{m}^{k}\right]$. In addition, the second row of (33) has two arguments for every cell in the first row. The first argument could be "1" or " 2 " depending on whether the cell in the first row belongs to $A^{k}$ or $B^{k}$, respectively. Moreover, the second argument for the second row in (33) could be $a_{n}^{k}$ or $b_{m}^{k}$; that is, $a_{1}^{k}=b_{1}^{k}=1, a_{2}^{k}=$ $b_{2}^{k}=2, \ldots$, and $a_{n}^{k}=n, b_{m}^{k}=m$, where $a_{n}^{k}$ and $b_{m}^{k}$ belong to $A^{k}$ and $B^{k}$, respectively.

To sort the matrix $C^{k}$ based on the 1st row is the second step which is obtained as

$$
C^{k}=\text { quicksort }\left(C^{k}, 1 \text { st row }\right) \text {. }
$$

The Quicksort algorithm then sorts the $C^{k}$ as

$$
C^{k}=\left[\begin{array}{cccc}
c_{1}^{k} & c_{2}^{k} & \cdots & c_{n+m}^{k} \\
\cdots & \cdots & \cdots & x_{n+m} y_{n+m}
\end{array}\right]_{2 \times(n+m)},
$$

where $c_{1}^{k}$ is minimum of the 1st row, $c_{n+m}^{k}$ is maximum of the 1st row, and $x_{n+m}$ is 1 or 2 to indicate that the value $c_{n+m}^{k}$
TABLE 5: CRI and SCRI schemes.

\begin{tabular}{ll}
\hline CRI & SCRI \\
\hline Implication & Implication \\
Cylindrical extension & Sorting program \\
Calculating relational matrix & Getting relational matrix \\
Aggregation & Aggregation \\
Composition & Composition \\
\hline
\end{tabular}

TABLE 6: CRI and SCRI time complexity comparison results.

\begin{tabular}{lcc}
\hline & $\begin{array}{c}\text { CRI } \\
\text { time complexity } \\
\text { (min operations) }\end{array}$ & $\begin{array}{c}\text { SCRI } \\
\text { time complexity } \\
\text { (min operations) }\end{array}$ \\
\hline Implication & $O(r n m)$ & $O\left(r(n+m) \log _{2}(n+m)\right)$ \\
\hline
\end{tabular}

belongs to $A^{k}$ or $B^{k}$, respectively. $y_{n+m}$ in (35) is used to specify the position of the value $c_{n+m}^{k}$ in $A^{k}$ or $B^{k}$ depending on whether $x_{n+m}$ is 1 or 2 , respectively. The second substep is getting relational matrix, which has $n+m$ steps. In the first step, consider the condition of $x_{n+m}$ as

$$
\begin{aligned}
& \text { if } x_{n+m}=1 \text {, then all values in the } y_{n+m} \text { th row of } R^{k} \text { are } \\
& \text { equal to } c_{n+m}^{k} \text {; } \\
& \text { if } x_{n+m}=2 \text {, then all values in the } y_{n+m} \text { th column of } \\
& R^{k} \text { are equal to } c_{n+m}^{k} \text {. }
\end{aligned}
$$

In the second step, consider the condition of $x_{n+m-1}$, and keep on continuing until $x_{1}$.

(3) CRI and SCRI Schemes. Table 5 showed the CRI and SCRI schemes. In the implication step, the main CRI operation is "calculating relational matrix" and the main SCRI operation is "sorting program."

(4) Time Complexity. SCRI is an improvement method from correcting the CRI implication step. The time complexity of the implication step for these two methods is compared and the results are shown in Table 6 . The same $r, n$, and $m$ parameters and the Quicksort are adopted for the SCRI sorting program. Following Theorem 1, the time complexity of an array with $(n+m)$ elements is $O\left((n+m) \log _{2}(n+m)\right)$. With SISO $r$-rule fuzzy system, the time complexity of the implication step of SCRI method is denoted as

$$
O\left(r(n+m) \log _{2}(n+m)\right) .
$$

Based on (30), the time complexity of the implication step for CRI method can be determined as

$$
O(r n m)
$$

The input dimension equals the output dimension in Figure 4 and the number of rules is 10 . The red line (curve for $\mathrm{CRI}$ ) shows the relation of $x$ (dimension of input and output) and $y$ (operations), and the blue line (dash curve for SCRI) shows the relation of $x$ (dimension of input and output) and $y$ (operations), respectively. Moreover, the green line shows a reference line with the input dimension as 40 and the output 
dimension as 40 . The red point in the red line with input dimension $=40$, output dimension $=40$, and the number of rules $=10$ shows CRI $=16000$ operations. The blue point at the blue line with input dimension $=40$, output dimension $=$ 40 , and number of rules $=10$ shows SCRI $=5058$ operations. It is clear to see that the CRI method time complexity is greater than the SCRI method time complexity.

(5) Space Complexity. In this subsection SCRI is discussed as an improvement in the CRI implication step. The implication step space complexity of the CRI and SCRI methods is shown in Table 7. By substituting $r, n$, and $m$ of the output elements into (30) for every relational matrix, the CRI needs a space of $O(\mathrm{~nm})$. With SISO $r$ rules the CRI needs a space of $O(\mathrm{rnm})$. Thus, based on (34), for every relational matrix, it is known that SCRI needs a space of $O(n+m)$. With SISO $r$ rules, SCRI needs a space of $O(\mathrm{rnm})$ to store $r$ relational matrixes.

Based on (34), for every relational matrix, SCRI just needs a space of $O(n+m)$ but applying (30) CRI needs a space of $O(n m)$.

\subsubsection{IRI and SIRI}

(1) IRI. The first step in the IRI method is the implication step as defined in Section 2.2.1(1), which includes two substeps. The first substep is cylindrical extension defined as

$$
A^{k} \_\mathrm{ex}=\left[\begin{array}{cccc}
a_{11}^{k} & a_{12}^{k} & \cdots & a_{1 m}^{k} \\
a_{21}^{k} & a_{22}^{k} & \cdots & a_{2 m}^{k} \\
\vdots & \vdots & \cdots & \vdots \\
a_{n 1}^{k} & a_{n 2}^{k} & \cdots & a_{n m}^{k}
\end{array}\right]_{n \times m},
$$

where $A^{k} \_$ex is extended by $A^{k}, a_{i j}^{k}=a_{i}^{k}$, with $A^{k}=\left[a_{i}^{k}\right]_{i=1}^{n}$, $i=1,2, \ldots, n, j=1,2, \ldots, m, k=1,2, \ldots, r$ :

$$
B^{k} \_\mathrm{ex}=\left[\begin{array}{cccc}
b_{11}^{k} & b_{12}^{k} & \cdots & b_{1 m}^{k} \\
b_{21}^{k} & b_{22}^{k} & \cdots & b_{2 m}^{k} \\
\vdots & \vdots & \cdots & \vdots \\
b_{n 1}^{k} & b_{n 2}^{k} & \cdots & b_{n m}^{k}
\end{array}\right]_{n \times m},
$$

where $B^{k}$ _ex is extended by $B^{k}, b_{i j}^{k}=b_{j}^{k}$, with $B^{k}=\left[b_{j}^{k}\right]_{j=1}^{m}$, $i=1,2, \ldots, n, j=1,2, \ldots, m, k=1,2, \ldots, r$.

The second substep is calculating relation matrix defined as

$$
R^{k}=\min \left(A_{-}^{k} \text { ex, } B_{-}^{k} \text { ex }\right)
$$

where $R^{k}$ is the relation matrix of rule $k$. The implication is "min" as Mamdani fuzzy model. The second step of IRI method is composition which is defined as

$$
B^{k^{\prime}}=A^{\prime} \circ R^{k}
$$

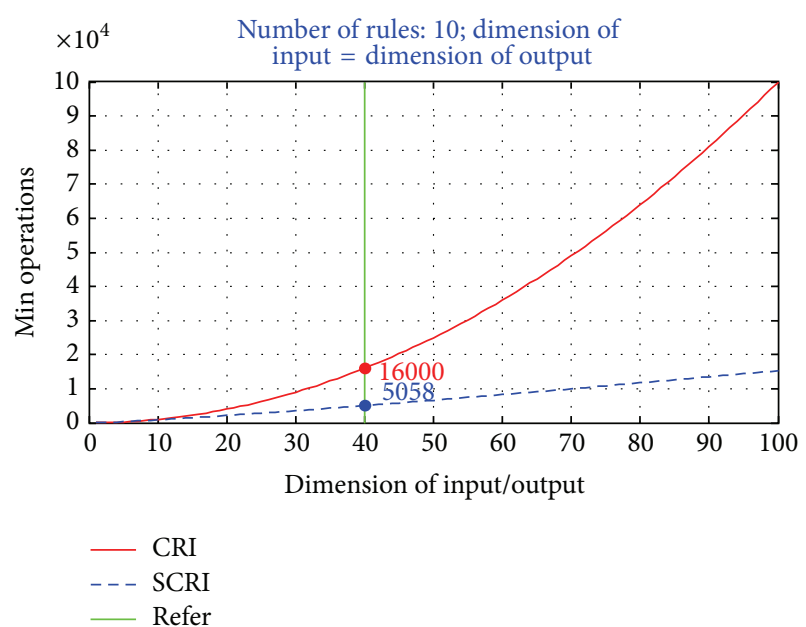

FIGURE 4: CRI and SCRI implication step time complexity comparison with $r=10, n=m$.

TABLE 7: CRI and SCRI space complexity comparison.

\begin{tabular}{ccccc}
\hline & $\begin{array}{c}\text { CRI } \\
\text { space complexity } \\
\text { (cells) }\end{array}$ & \multicolumn{2}{c}{$\begin{array}{c}\text { SCRI } \\
\text { space complexity } \\
\text { (cells) }\end{array}$} \\
\hline \multirow{3}{*}{ Implication } & $\begin{array}{c}\text { 1 relation } \\
\text { matrix } \\
r \text { relation }\end{array}$ & $O(n m)$ & $\begin{array}{c}1 \text { relation } \\
\text { matrix }\end{array}$ & $O(n+m)$ \\
matrixes & $O(r n m)$ & $\begin{array}{c}r \text { relation } \\
\text { matrixes }\end{array}$ & $O(\mathrm{rnm})$ \\
\hline
\end{tabular}

where $B^{k^{\prime}}$ is the inference result of rule $k$ and it is calculated by composing observation $A^{\prime}$ with the relational matrix $R^{k}$ of rule $k$ and $\circ$ represents the composition method. The third step of IRI is aggregation defined as

$$
B^{\prime}=\bigcup_{k=1}^{r} B^{k^{\prime}}
$$

where $B^{\prime}$ is the final inference result. It is calculated by combining all of the inference results $B^{k^{\prime}}$ with $k=1,2, \ldots, r$.

(2) SIRI (Sort IRI). Containing three steps for SIRI method, the first step is implication which involves two substeps. The first substep is sorting program and the second substep is getting relational matrix. The second step is composition defined as $B^{k^{\prime}}=A^{\prime} \circ R^{k}$, and the third one is aggregation defined as $B^{\prime}=\bigcup_{k=1}^{r} B^{k^{\prime}}$.

Furthermore, the SIRI implication has two more substeps. The first substep is sorting program. The matrix $C^{k}$ is generated as

$$
C^{k}=\left[\begin{array}{cccccccc}
a_{1}^{k} & a_{2}^{k} & \cdots & a_{n}^{k} & b_{1}^{k} & b_{2}^{k} & \cdots & b_{m}^{k} \\
1.1 & 1.2 & \cdots & 1 . n & 2.1 & 2.2 & \cdots & 2 . m
\end{array}\right]_{2 \times(n+m)},
$$

where the elements in the first row of (43) are the combination of $A^{k}$ and $B^{k}$ with $A^{k}=\left[a_{1}^{k}, a_{2}^{k}, \ldots, a_{n}^{k}\right], B^{k}=\left[b_{1}^{k}, b_{2}^{k}, \ldots, b_{m}^{k}\right]$. In addition, the second row of (43) has two arguments for 
TABLE 8: IRI and SIRI schemes.

\begin{tabular}{ll}
\hline IRI & SIRI \\
\hline Implication & Implication \\
Cylindrical extension & Sorting program \\
Calculating relational matrix & Getting relational matrix \\
Composition & Composition \\
Aggregation & Aggregation \\
\hline
\end{tabular}

every cell in the first row, and the first argument could be " 1 " or " 2 " depending on the cell of the first row belonging to $A^{k}$ or $B^{k}$, respectively. Moreover, the second argument of the second row in (43) could be $a_{n}^{k}$ or $b_{m}^{k}$; that is, $a_{1}^{k}=b_{1}^{k}=1$, $a_{2}^{k}=b_{2}^{k}=2, \ldots$, and $a_{n}^{k}=n, b_{m}^{k}=m$, where $a_{n}^{k}$ and $b_{m}^{k}$ belong to $A^{k}$ and $B^{k}$, respectively.

Sort the matrix $C^{k}$ based on the 1st row and obtained as

$$
C^{k}=\text { quicksort }\left(C^{k}, 1 \text { st row }\right) \text {. }
$$

After taking the Quicksort algorithm, the final $C^{k}$ is sorted as

$$
C^{k}=\left[\begin{array}{cccc}
c_{1}^{k} & c_{2}^{k} & \cdots & c_{n+m}^{k} \\
\cdots & \cdots & \cdots & x_{n+m} y_{n+m}
\end{array}\right]_{2 \times(n+m)},
$$

where $c_{1}^{k}$ is minimum of the 1st row and $c_{n+m}^{k}$ is maximum of the 1st row and $x_{n+m}$ is 1 or 2 to indicate the value $c_{n+m}^{k}$ belonging to $A^{k}$ or $B^{k}$, respectively. $y_{n+m}$ in (45) is used to define the position of the value $c_{n+m}^{k}$ in $A^{k}$ or $B^{k}$ depending on $x_{n+m}$ which is 1 or 2 , respectively. The second substep is getting relational matrix, which has $n+m$ steps. In the first step, let us consider the condition of $x_{n+m}$ as

$$
\begin{aligned}
& \text { if } x_{n+m}=1 \text {, then all values in the } y_{n+m} \text { th row of } R^{k} \text { are } \\
& \text { equal to } c_{n+m}^{k} \text {; } \\
& \text { if } x_{n+m}=2 \text {, then all values in the } y_{n+m} \text { th column of } \\
& R^{k} \text { are equal to } c_{n+m}^{k} .
\end{aligned}
$$

In the next step, consider the condition of $x_{n+m-1}$, and keep on the algorithm until $x_{1}$.

(3) IRI and SIRI Schemes. The main IRI operation is "calculating relational matrix" and the main operation of the SIRI belongs to "sorting program." The operation schemes of IRI and SIRI are listed in Table 8.

(4) Time Complexity. The comparison results are shown in Table 9. Three parameters $r, n$, and $m$ and Quicksort are used for the SIRI sorting program to verify the time complexity. The time complexity of an array with $(n+m)$ elements is $O((n+$ $\left.m) \log _{2}(n+m)\right)$ which can be determined from Theorem 1 again. With SISO $r$-rule fuzzy systems, the time complexity of the implication step of SIRI method is

$$
O\left(r(n+m) \log _{2}(n+m)\right) .
$$

Furthermore, the time complexity of the implication step of IRI method can be obtained from (40) and expressed as

$$
O(r n m) \text {. }
$$

TABLE 9: Comparison of the time complexity of IRI and SIRI.

\begin{tabular}{lcc}
\hline & $\begin{array}{c}\text { IRI } \\
\text { time complexity } \\
\text { (min operations) }\end{array}$ & $\begin{array}{c}\text { SIRI } \\
\text { time complexity } \\
\text { (min operations) }\end{array}$ \\
\hline Implication & $O(r n m)$ & $O\left(r(n+m) \log _{2}(n+m)\right)$ \\
\hline
\end{tabular}

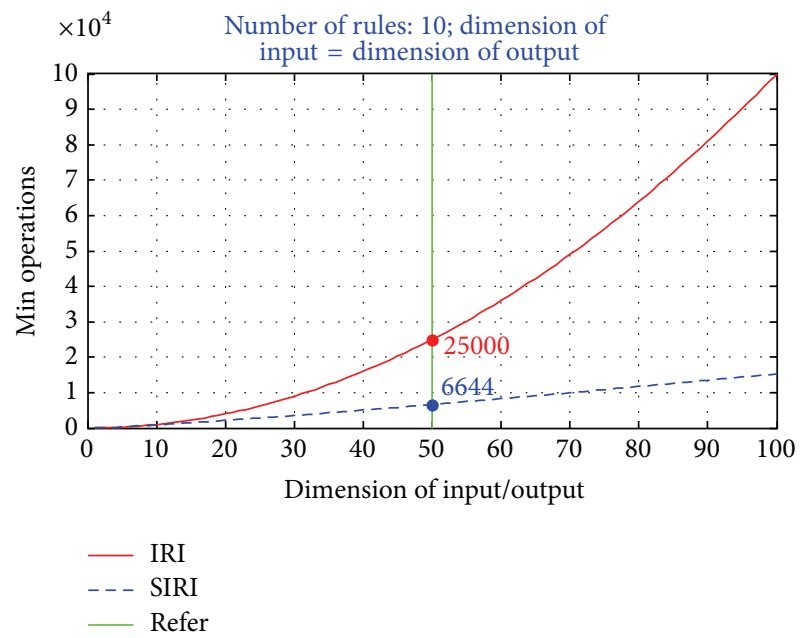

FIGURE 5: IRI and SIRI implication step time complexity comparison results with $r=10, n=m$.

In order to understand the operation complexity of these methods, the results from time complexity comparison are shown in Figure 5. The input dimension is considered equal to the output dimension. The number of rules is set to 10 . Red line (curve for IRI) shows the relation of $x$ (dimension of input and output) and $y$ (operations). On the contrary, the blue line (dash curve for SIRI) represents the relation of $x$ (dimension of input and output) and $y$ (operations). On the other hand, the green line is a reference line which with the input dimension is 50 and the output dimension is 50 . Red point, at the red line with input dimension $=50$, output dimension $=50$, number of rules $=10$, shows operations of IRI $=25000$ operations. Blue point, at the blue line with input dimension $=50$, output dimension $=50$, and number of rules $=10$, showed operations of SIRI $=6644$ operations. As previously described, it is clear that the IRI operations are definitely larger than the SIRI operations.

(5) Space Complexity. In this subsection, the space complexity of the SIRI implication step is shown. This is an improvement in the IRI implication step. In Table 10, the space complexity of IRI and SIRI is illustrated with the $r, n$, and $m$ parameters again. By taking account of every relational matrix into (40), the space complexity of $\mathrm{O}(\mathrm{nm})$ is for IRI; on the contrary, with SISO $r$ rules the IRI requires space of $O(\mathrm{rnm})$.

Based on (44) for every relational matrix, SIRI just needs a space of $O(n+m)$ but applying (40) IRI needs a space of $O(n m)$. 


\section{Conclusion}

The SCRI and SIRI schemes for improving CRI and IRI operation performance for the fuzzy control theoretical approach are proposed in this paper. The previous methods were proven to reduce the time and space complexity for fuzzy expert systems by improving the most important step (implication). It was shown that the time consuming, for example, the implication step, needs an operation of $O(n \times m)$, in the traditional methods with compositional rule-based inference (CRI) and individual rule-based inference (IRI) will be significantly decreased as $O\left((n+m) \log _{2}(n+m)\right)$. With SISO multirule systems the two SCRI and SIRI schemes are proven to perform much better than CRI and IRI in time and space complexity. This paper generated better SCRI (Sort CRI) and SIRI (Sort IRI) performance in which two can replace the traditional CRI and IRI using the Mamdani implication, respectively. We are going to extend these results for MISO and MIMO fuzzy systems in the future works.

\section{Appendices}

\section{A. Quicksort Proof}

Consider

$$
\begin{aligned}
& C(l)=l+\left(\frac{2}{l}\right) \sum_{i=0}^{l-1} C(i) \\
& \Longleftrightarrow l C(l)=l^{2}+2 \sum_{i=0}^{l-1} C(i) \\
& \Longleftrightarrow(l-1) C(l-1)=(l-1)^{2}+2 \sum_{i=0}^{l-2} C(i) \\
& \Longleftrightarrow l C(l)-(l-1) C(l-1)=l^{2}-(l-1)^{2}+2 C(l-1) \\
& \Longleftrightarrow l C(l)=(l+1) C(l-1)+2 l-1 .
\end{aligned}
$$

\section{B. Composition Step}

Consider

$$
\begin{aligned}
B^{\prime} & =A^{\prime} \circ R \\
& =\sup \min \left(A^{\prime} \_ \text {ex, } R\right) \\
& =\sup \left(\min \left(A^{\prime} \_ \text {ex, } R\right)\right) \\
& =\sup \left[\begin{array}{cccc}
b_{11}^{\prime} & b_{12}^{\prime} & \cdots & b_{1 m}^{\prime} \\
b_{21}^{\prime} & b_{22}^{\prime} & \cdots & b_{2 m}^{\prime} \\
\vdots & \vdots & \cdots & \vdots \\
b_{n 1}^{\prime} & b_{n 2}^{\prime} & \cdots & b_{n m}^{\prime}
\end{array}\right]_{n \times m}=\left[\begin{array}{llll}
b_{1}^{\prime} & b_{2}^{\prime} & \cdots & b_{m}^{\prime}
\end{array}\right],
\end{aligned}
$$

TABLE 10: IRI and SIRI space complexity comparison.

\begin{tabular}{ccccc}
\hline & \multicolumn{1}{c}{$\begin{array}{c}\text { IRI } \\
\text { space complexity } \\
\text { (cells) }\end{array}$} & $\begin{array}{c}\text { SIRI } \\
\text { space complexity } \\
\text { (cells) }\end{array}$ \\
\hline \multirow{3}{*}{ Implication } & $\begin{array}{c}\text { 1 relation } \\
\text { matrix } \\
r \text { relation } \\
\text { matrixes }\end{array}$ & $O(\mathrm{~nm})$ & $\begin{array}{c}\text { 1 relation } \\
\text { matrix }\end{array}$ & $O(n+m)$ \\
& & $\begin{array}{c}r \text { relation } \\
\text { matrixes }\end{array}$ & $O(\mathrm{rnm})$ \\
\hline
\end{tabular}

where

$$
A^{\prime} \_\mathrm{ex}=\left[\begin{array}{cccc}
a_{11}^{\prime} & a_{12}^{\prime} & \cdots & a_{1 m}^{\prime} \\
a_{21}^{\prime} & a_{22}^{\prime} & \cdots & a_{2 m}^{\prime} \\
\vdots & \vdots & \cdots & \vdots \\
a_{n 1}^{\prime} & a_{n 2}^{\prime} & \cdots & a_{n m}^{\prime}
\end{array}\right]_{n \times m}
$$

and $a_{i j}^{\prime}=a_{i}^{\prime}$ with $A^{\prime}=\left[a_{i}^{\prime}\right]_{i=1}^{n}$,

$$
\begin{gathered}
R=\left[\begin{array}{cccc}
q_{11} & q_{12} & \cdots & q_{1 m} \\
q_{21} & q_{22} & \cdots & q_{2 m} \\
\vdots & \vdots & \cdots & \vdots \\
q_{n 1} & q_{n 2} & \cdots & q_{n m}
\end{array}\right]_{n \times m}, \\
q_{i j}=\bigcup_{k=1}^{r} q_{i j}^{k}, b_{i j}^{\prime}=\min \left(a_{i j}^{\prime}, q_{i j}\right), b_{j}^{\prime}=\bigcup_{i=1}^{n} b_{i j}^{\prime}, i= \\
1,2, \ldots, n, j=1,2, \ldots, m .
\end{gathered}
$$

\section{Conflict of Interests}

The authors declare that there is no conflict of interests regarding the publication of this paper.

\section{References}

[1] L. A. Zadeh, "Outline of new approach to the analysis of complex systems and decision processes," IEEE Transactions on Systems, Man, and Cybernetics, vol. SMC-3, pp. 28-44, 1973.

[2] D. Dubois and H. Prade, "Fuzzy sets in approximate reasoning, part 1: inference with Possibility distributions," Fuzzy Sets and Systems, vol. 40, no. 1, pp. 143-202, 1991.

[3] I. B. Türken and Y. Tian, "How to select combination operators for fuzzy expert systems using CRI," in Proceedings of the North American Fuzzy Logic Processing Society (NAFIPS '92), vol. 1, pp. 29-38, Puerto Vallarta, Mexico, 1992.

[4] S. Assilian, Aritificial intelligence in the control of real dynamic systems [Ph.D. thesis], Queen Mary College, University of London, 1974.

[5] D. Dubois, "The role of fuzzy sets in decision sciences: old techniques and new directions," in Proceedings of the EUROFUSE Workshop Preference Modelling and Decision Analysis, Pamplona, Spain, 2010.

[6] B. Lazzerini and F. Marcelloni, "Reducing computation overhead in MISO fuzzy systems," Fuzzy Sets and Systems, vol. 113, no. 3, pp. 485-496, 2000. 
[7] K. Demirli and I. B. Turksen, "Rule break up with compositional rule of inference," in Proceedings of the IEEE International Conference on Fuzzy Systems, pp. 949-956, March 1992.

[8] E. H. Mamdani, "Application of fuzzy logic to approximate reasoning using linguistic synthesis," IEEE Transactions on Computers, vol. C-26, no. 12, pp. 1182-1191, 1977.

[9] M. Shahjalal, A. Sultana, N. K. Mitra, and A. F. M. Khodadad Khan, "Compositional rule of inference and adaptive fuzzy rule based scheme with applications," Annals of Pure and Applied Mathematics, vol. 3, no. 2, pp. 155-168, 2013.

[10] F. Liu, H. Geng, and Y.-Q. Zhang, "Interactive fuzzy interval reasoning for smart web shopping," Applied Soft Computing Journal, vol. 5, no. 4, pp. 433-439, 2005.

[11] I. Iancu, "A Mamdani type fuzzy logic controller," in Fuzzy Logic-Controls, Concepts, Theories and Applications, InTech, 2012.

[12] Z. Zheng, S. Wu, W. Liu, and K.-Y. Cai, "A feedback based CRI approach to fuzzy reasoning," Applied Soft Computing Journal, vol. 11, no. 1, pp. 1241-1255, 2011.

[13] J. Villar, "Conditions for equivalence betwen the compositional rule of infernce and compatibility modification inference," Fuzzy Systems, vol. 1, pp. 444-449, 1996.

[14] G.-J. Wang, "On the logic foundation of fuzzy reasoning," Information Sciences, vol. 117, no. 1-2, pp. 47-88, 1999.

[15] S. Song, C. Feng, and E. S. Lee, "Triple I method of fuzzy reasoning," Computers \& Mathematics with Applications, vol. 44, no. 12, pp. 1567-1579, 2002.

[16] D. Pei, "Formalization of implication based fuzzy reasoning method," International Journal of Approximate Reasoning, vol. 53, no. 5, pp. 837-846, 2012.

[17] G. Wang and J. Duana, "On robustness of the full implication triple I inference method with respect to finer measurements," International Journal of Approximate Reasoning, vol. 55, no. 3, pp. 787-796, 2014.

[18] Y. Tang and X. Yang, "Symmetric implicational method of fuzzy reasoning," International Journal of Approximate Reasoning, vol. 54, no. 8, pp. 1034-1048, 2013.

[19] M. Luo and N. Yao, "Triple I algorithms based on SchweizerSKLar operators in fuzzy reasoning," International Journal of Approximate Reasoning, vol. 54, no. 5, pp. 640-652, 2013.

[20] D. Pei, "Unified full implication algorithms of fuzzy reasoning," Information Sciences, vol. 178, no. 2, pp. 520-530, 2008.

[21] Y. Liu and M. Zheng, "The dual triple I methods of FMT and IFMT," Mathematical Problems in Engineering, vol. 2014, Article ID 507401, 8 pages, 2014.

[22] J. Tick and J. Fodor, "Fuzzy implications and inference processes," Computing and Informatics, vol. 24, no. 6, pp. 591-602, 2005.

[23] A. Kaur and A. Kaur, "Comparison of Mamdani-type and Sugeno-type fuzzy inference systems for air conditioning system," International Journal of Soft Computing and Engineering, vol. 2, no. 2, pp. 323-325, 2012.

[24] K. Guney and N. Sarikaya, "Comparison of Mamdani and Sugeno fuzzy inference system models dor resonant frequency calculation of rectangular microstrip antennas," Progress In Electromagnetics Research B, vol. 12, pp. 81-104, 2009.

[25] I. Elamvazuthi, P. Vasant, and J. Webb, “The application of Mamdani fuzzy model for auto zoom function of a digital camera," International Journal of Computer Science and Information Security, vol. 6, no. 3, pp. 126-134, 2009. 

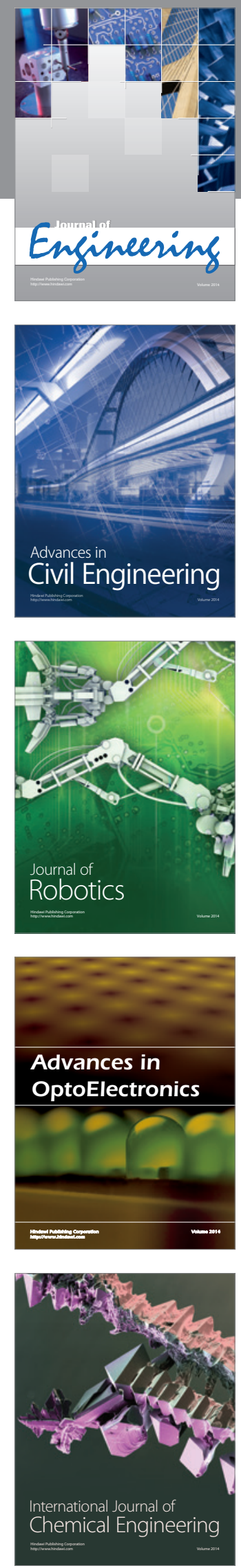

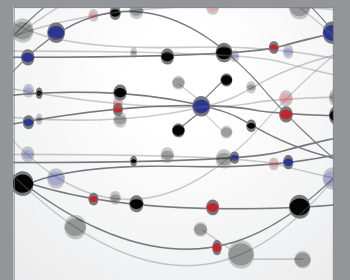

The Scientific World Journal
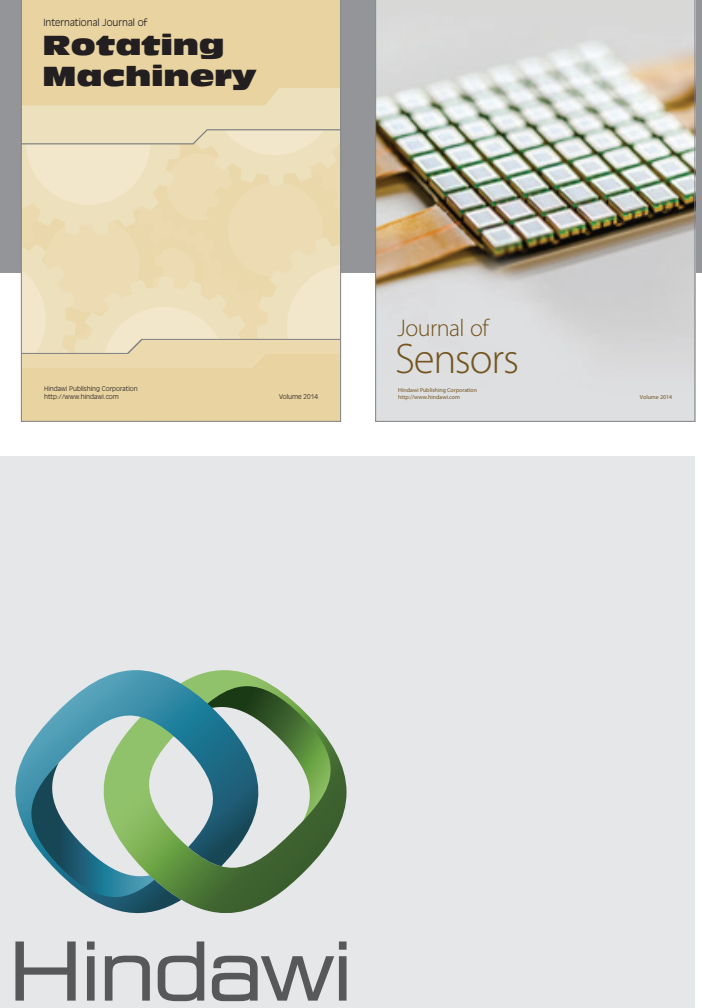

Submit your manuscripts at http://www.hindawi.com
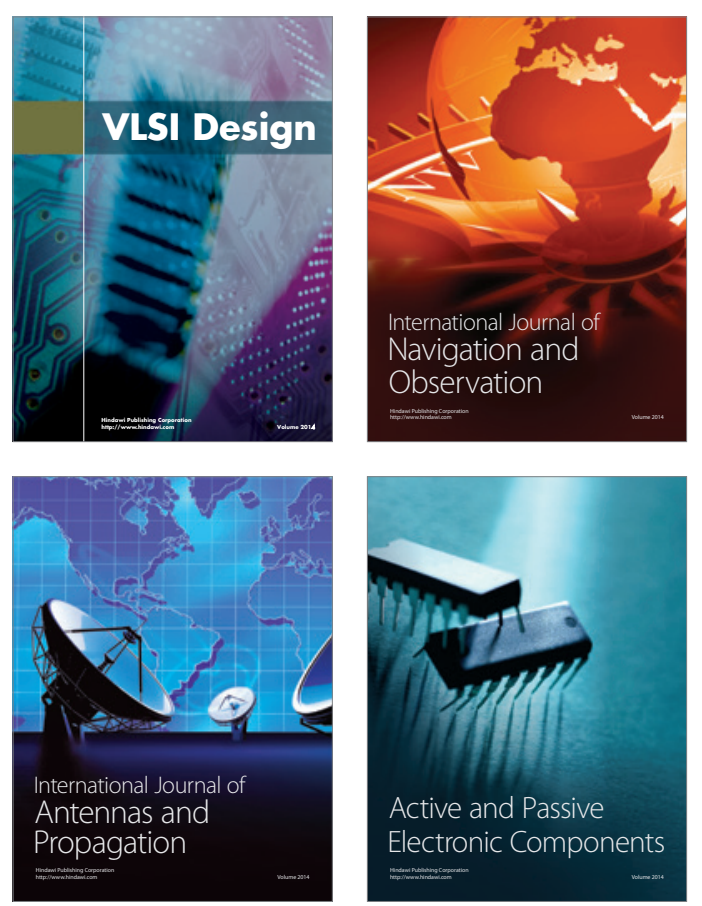
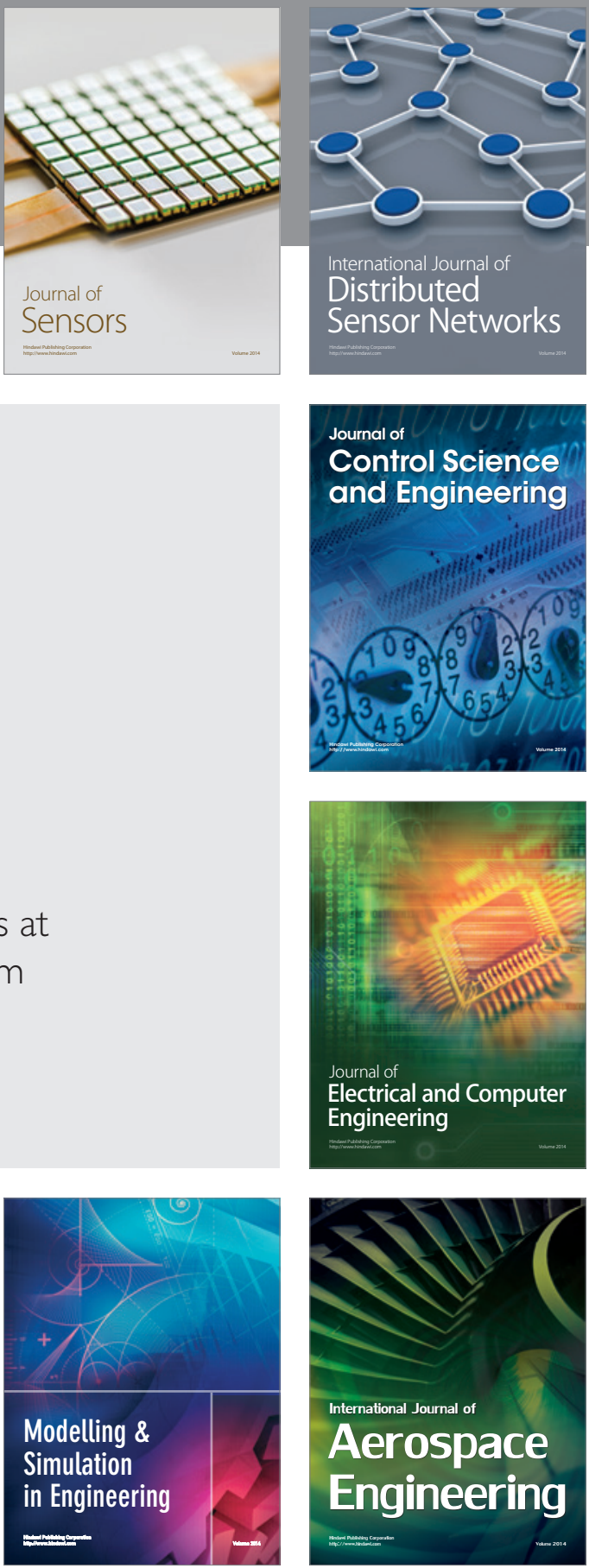

Journal of

Control Science

and Engineering
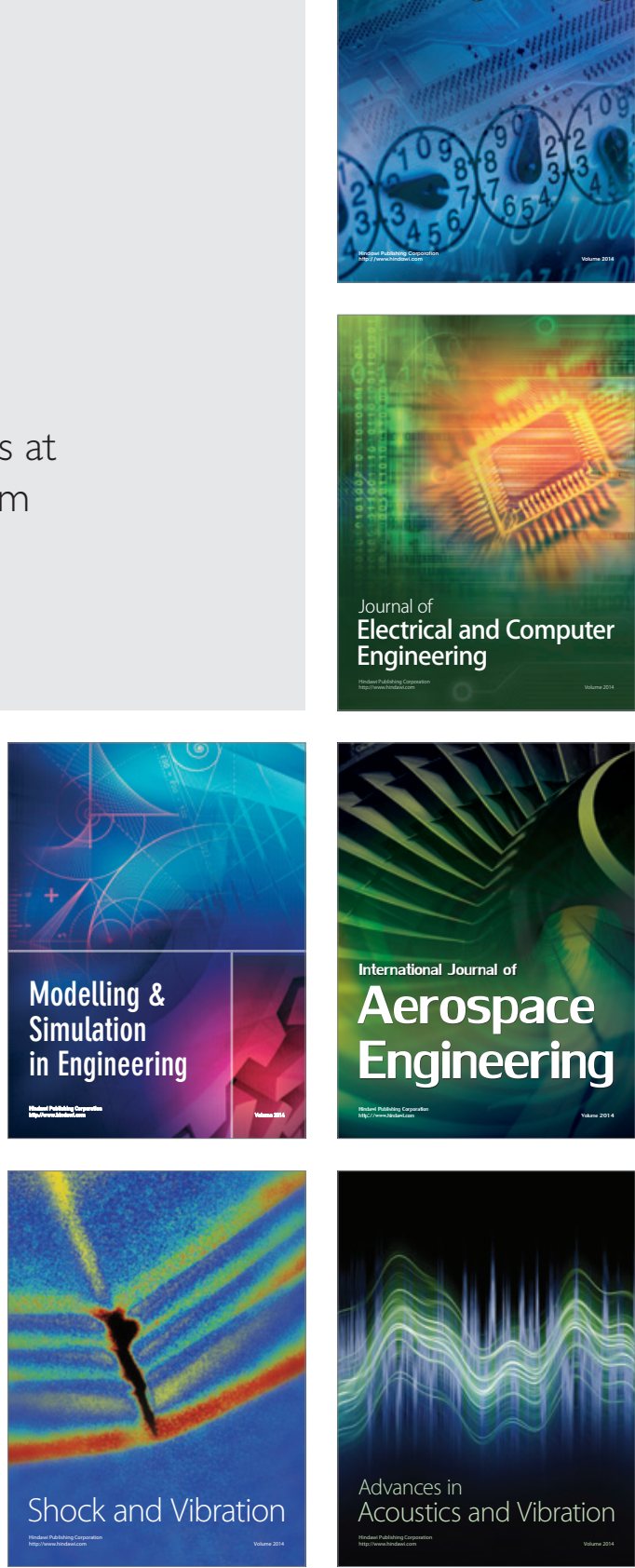